\title{
MANAGEMENT OF A CASE OF INTERTROCHANTERIC FRACTURE IN A 98 YEARS OLD PATIENT
}

\author{
Bhavna Royzada1 , Aditya Bidwaikar², Avijit Royzada ${ }^{3}$
}

${ }^{1}$ Associate Professor, Department of Anaesthesiology and Critical Care, Chhattisgarh Institute of Medical Sciences, Chhattisgarh, India. ${ }^{2}$ Senior Resident, Department of Anaesthesiology and Critical Care, Chhattisgarh Institute of Medical Sciences, Chhattisgarh, India. 3Physician \& Director, Department of Anaesthesiology and Critical Care, Saibaba Hospital, Bilaspur, Chattisgarh, India.

HOW TO CITE THIS ARTICLE: Royzada B, Bidwaikar A, Royzada A. Management of a case of intertrochanteric fracture in a 98 years old patient. J. Evolution Med. Dent. Sci. 2019;8(18):1519-1520, DOI: 10.14260/jemds/2019/337

\section{PRESENTATION OF CASE}

A 98 years old male patient, diagnosed as intertrochanteric fracture femur, was posted for fixation by dynamic hip screw. His weight was $70 \mathrm{Kg}$, height $160 \mathrm{~cm}$, blood pressure of $130 / 90 \mathrm{~mm}$ of $\mathrm{Hg}$ and a pulse of 56 per minute. He was conscious and oriented in time, place and person.

There was no history of chest pain or dyspnoea. His investigations revealed haemoglobin, creatinine, TLC, DLC, with in normal range. Serum sodium $132 \mathrm{mEq} / \mathrm{litre}$. His random blood sugar was $94 \mathrm{mg} \%$. Chest x-ray was showing mild emphysematous changes. Electrocardiogram showed ST segment depression of $1 \mathrm{~mm}$ and $\mathrm{T}$ wave inversion in chest leads V 1\& V2. Echocardiography was normal with LVEF $42 \%$.

In view of extreme old age and low ejection fraction, epidural anaesthesia was planned. Procedure was explained to the patient and he was kept nil by mouth for 8 hours. Patient was premedicated with inj. Metoclopramide $10 \mathrm{mg}$ half an hour before surgery. Intravenous access was secured with 18G cannula and monitor was attached. ECG, blood pressure, Sp02, respiratory rate and temperature was monitored.

Patient was placed in sitting position with legs hanging by the side of the operation table \& supported on a footrest and 18G Tuohy needle was inserted by a paramedian approach into L4-L5 interspace using loss of resistance technique. Epidural catheter was introduced through needle up to $5 \mathrm{~cm}$ in cephalad direction. ${ }^{3}$ Test dose of $2 \mathrm{ml}$ of lidocaine with adrenaline was given, after that drug solution $8 \mathrm{ml}$ lidocaine $2 \%+6 \mathrm{ml}$ of $0.5 \%$ Bupivacaine $+50 \mathrm{mg}$ Tramadol) was slowly injected through catheter after negative aspiration. T8 sensory level was achieved and procedure lasted for one and half hour. Intravascular volumes were maintained by giving Ringer lactate and normal saline solutions. Patient remained hemodynamically stable throughout the procedure except two initial episodes of hypotension which were managed by inj. Mephentermine $3 \mathrm{mg}$. There was no significant blood loss during the surgery. ${ }^{4}$ No untoward complication occurred throughout the course of operation. Patient was shifted to intensive therapy unit for postoperative care. Eight hourly supplementations of Inj. Tramadol $100 \mathrm{mg}$ was done through epidural catheter for 3 days for postoperative analgesia. Patient did well postoperatively and was discharged from hospital after one week.

'Financial or Other Competing Interest': None.

Submission 14-03-2019, Peer Review 18-04-2019,

Acceptance 26-04-2019, Published 06-05-2019.

Corresponding Author:

Dr. Bhavna Royzada,

Saibaba Hospital,

Gaurav Path, Bilaspur, Chhattisgarh, India.

E-mail: bhavanathegreat@Yahoo.co.in

DOI: $10.14260 /$ jemds $/ 2019 / 337$

\section{(c) (i) $(9)$}

\section{DISCUSSION}

Advances in anaesthetic and surgical techniques and perioperative care have substantially reduced the associated risks. However, overall mortality in the general population remains at $1.2 \%$, \& those over 80 yrs., of age has morbidity of $51 \%$ mortality $7 \% .^{1}$

Among the steadily increasing population of surgical patients aged 65 yrs., and above, patients aging 90 years and above are also on increasing trend. As a result, greater number of patients are presenting for surgery with agerelated, pre-existing conditions that place them at greater risk of an adverse outcome, such as cardiac or pulmonary disease or diabetes mellitus. It is therefore, not surprising that the elderly have highest mortality rate in the adult surgical population. Postoperative adverse effects on the cardiac, pulmonary, cerebral systems, and on cognitive functions are the main concerns for elderly surgical patients.

There are various choices like general anaesthesia, spinal, epidural, regional techniques out of this, epidural anaesthesia is safer in comparison to other techniques ${ }^{2}$. More haemodynamic stability and good post-operative analgesia are the main advantages of epidural anaesthesia. Although, age related risk is always a concern

Giving anaesthesia to elderly patient is always a challenge for an anaesthetist. Perioperative morbidity and mortality in elderly continues to be an important problem due to their low cardiac and pulmonary reserve. Elderly patients have age related osteoporosis, osteoarthritis, joint stiffness etc, so they are prone to fractures with minimal injuries. We have reported this case of 98-year-old patient as surgeries in this age group are rarely encountered. Epidural anaesthesia has a distinct advantage over general anaesthesia in terms of improved pulmonary and myocardial function and postoperative analgesia. Early mobility in post-operative period is beneficial in reducing the chances of thromboembolism and hastens recovery. Hence, choice of anaesthesia plays a very important role in post-operative morbidity and mortality.

Hypertension and dyspnoea are the most important risk factors in patients aged 80 years and above. Increased ASA status, perioperative transfusion, emergency operation and weight loss are responsible for postoperative morbidity. ${ }^{5} \mathrm{~A}$ 30 minute increment in operative time increases odds of mortality by $17 \%$ in patients older than 80 years of age.

Regional anaesthesia offers several advantages to elderly patients including provision of postoperative analgesia with minimal side effects. Neuraxial anaesthesia may reduce the incidence of thromboembolic complications in geriatric patients, especially following orthopaedic and lower extremity vascular surgery. ${ }^{6}$ Epidural anaesthesia requires cooperative patient who can lie still for the duration of surgery. Epidural analgesia reduces incidence of postoperative atelectasis and pulmonary infection as compared to general anaesthesia in patients over 80 years of 
age. Although knowledge of physiology of aging should help reduce age related complications, unpredictable physiological and pharmacological interactions and diagnostic difficulties are unavoidable.

Epidural anaesthesia attenuates perioperative stress response, improves myocardial oxygenation, it also inhibits endocrine and inflammatory responses with protein catabolism. One major advantages of Epidural anaesthesia is the lower incidence of postoperative thromboembolism due to peripheral vasodilatation and the maintenance of venous blood flow in lower extremities. Post-operative mental status is maintained, 7 unlike the undesired reduction after General anaesthesia.

In this case epidural anaesthesia with lidocaine and Bupivacaine resulted in satisfactory anaesthesia with favourable effect on myocardium without morbidity even in extremes of age.

Epidural anaesthesia facilitates early mobilization, improves convalescence, and reduces morbidity with decreased hospital stay. It should be considered as a reliable anaesthetic technique as compared to general anaesthesia for surgical procedures in elderly.

\section{REFERENCES}

[1] Turrentine FE, Wang H, Simpson VB, et al. Surgical risk factors, morbidity and mortality in elderly patients. J Am Coll Surg 2006;203(6):865-77.

[2] Chung F, Meier R, Lautenschlager E, et al. General or spinal anaesthesia: which is better in elderly? Anaesthesiology 1987;67(3):422-7.

[3] Murakawa T, Anzawa N, Hashimoto Y, et al. Anaesthetic management of elderly patients aged 90 years or older. Masui 2004;53(2):167-72.

[4] Tsui BC, Wagner A, Finucane B. Regional anaesthesia in the elderly: a clinical guide. Drugs and Aging 2004;21(14):895-910.

[5] Hamel MB, Henderson WG, Khuri SF, et al. Surgical outcomes for patients aged 80 and older: morbidity and mortality from major noncardiac surgery. Journal of American Geriatrics Society 2005;53(3):424-9.

[6] Novak-Jankovic V. Regional anaesthesia for the elderly patients. Periodicum Biologorum 2013;115(2):119-23.

[7] Chung FF, Chung A, Meier RH, et al. Comparison of perioperative mental function after general anaesthesia and spinal anaesthesia with intravenous sedation. Canadian Journal of Anaesthesia 1989;36(4):382-7. 